'Escuela de Medicina, Universidad de Valparaíso, Valparaíso-Chile. ${ }^{2}$ Instituto de Políticas Públicas en Salud, Universidad San Sebastián, Santiago-Chile. Escuela de Sociología Universidad de Valparaíso, Valparaíso-Chile. aEgresado de Sociología.

Fuente de apoyo financiero: Proyecto de investigación financiado por la Vicerrectoría de Investigación de la Universidad San Sebastián, № 2012-0006-R La institución no tuvo injerencia en el diseño ni la ejecución del estudio.

Recibido el 31 de julio de 2014, aceptado el 11 de marzo de 2015.

Correspondencia a: Mario Parada L. Hontaneda 2653, cuarto piso, Valparaíso, Chile. mario.parada@uv.cl.

\section{Perfiles de egreso de las carreras de Medicina en Chile}

\author{
MARIO PARADA, MARÍA I. ROMERO², FABIÁN MORAGA3,a
}

\section{Professional profiles of graduates from Chilean Faculties of Medicine}

Background: The professional profile of health care professionals should incorporate recommendations of international agencies and adapt to the local conditions of each country. Aim: To conduct a qualitative analysis of Medical Graduate Profiles of universities grouped in the Chilean Association of Medicine Faculties (ASOFAMECH), characterizing its Social Focus, Humanist Approach, Social and Communication Skills. Material and Methods: Documentary analysis of profiles published on the respective web pages, using Atlas Ti software, establishing emerging categories and subcategories. These profiles were compared with the recommendations of the Pan-American Health Organization. Results: Data in Social Focus suggests that although community issues are a common element, the work in primary health and health promotion are rarely included. The Humanist Approach is addressed more commonly than the Social Focus, emphasizing humanization of care, ethical and religious values. Although, social and communication skills are scarcely acknowledged, those mentioned are teamwork and leadership role. Conclusions: There is a marked heterogeneity in the information declared and universities have not fully incorporated the recommendations of international organizations.

(Rev Med Chile 2015; 143: 512-519)

Key words: Education, Medical; Primary health care; Schools, medical; Undergraduate.

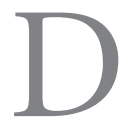
esde que Harden demostró que la formación médica se encuentra centrada en disciplinas parceladas y en la atención hospitalaria, la discusión sobre la formación generalista es importante ${ }^{1,2}$ : algunos autores abogan por un modelo emergente de educación médica para Latinoamérica, caracterizado por un dominio selectivo de los conocimientos básicos, por la comprensión de la conducta biopsicosocial, prevención y la salud comunitaria en igual importancia que los aspectos curativos ${ }^{3,4}$. Se ha abogado por introducir las ciencias sociales en la educación médica, siendo la principal tarea del pregrado exponer la determinación de la salud ${ }^{5}$.

En Chile, la formación médica con contenidos de Ciencias Sociales y Humanas es comentada en varios artículos ${ }^{6-13}$. Goic et al., cuantificaron la formación "humanística y psicosocial" en 5,4\% del total de la formación ${ }^{14}$. El último estudio publicado evidencia que la Universidad de Valparaíso tiene $9 \%$ de las horas destinadas a Ciencias Sociales y Humanas ${ }^{15}$.

La actual Reforma de Salud ha contemplado el fortalecimiento del nivel primario mediante la conformación de equipos multidisciplinarios, incorporación del modelo biopsicosocial, y profundización de la promoción y prevención ${ }^{16}$.

La Academia Chilena de Medicina recomienda que el perfil del egresado considere formación ética, humanística y en investigación como ejes curriculares ${ }^{17}$. 
Se ha planteado una misión social de la educación médica, lo que implica integrar contenidos biomédicos con sociales-humanos ${ }^{18}$. Para la OPS -Organización Panamericana de la Salud-, la salud va más allá de la medicina curativa, y se debe incorporar la visión de los determinantes sociales $^{19,20}$.

Estos planteamientos están en la redefinición de la formación médica propuesta por OPS: basada en la comunidad, orientación generalista, contenidos de salud pública, salud familiar y comunitaria, con enfoque de atención primaria en salud (APS), permitiendo un pensamiento interdisciplinario y comportamiento ético ${ }^{18,19-22}$. La política pública en Chile ha seguido históricamente las recomendaciones OPS, por ende es esperable que acoja, a lo menos parcialmente, estas orientaciones.

En Chile, el aumento de los titulados de medicina ha sido evidente ${ }^{23}$, y la tendencia de los últimos años apunta hacia un predominio de los médicos especialistas ${ }^{24,25}$. Algunos factores que explican aquello son la inadecuación de la carrera funcionaria en APS, influencia de la educación médica, y carencia de un adecuado perfil de egreso ${ }^{26,27}$.

Existe la convicción de que la mayor parte de los pregrados de medicina centran su enseñanza en el ámbito hospitalario, con marcado acento en la especialización ${ }^{27}$, a pesar de que varias escuelas llevan a cabo reformas curriculares tendientes mejorar las falencias s-9,14.28-31. $^{\text {. }}$

Se apunta a que los médicos en formación no están preparados para realizar una acción óptima en APS, ni dar respuesta a las necesidades generadas por la reforma ${ }^{27,32,33}$.

ASOFAMECH (asociación de las facultades de medicina de Chile), plantea que los desafíos de la educación médica son, entre otros, el fortalecimiento de la vocación, profesionalismo médico y un perfil humanista ${ }^{34}$. Recordando al Dr. Halfdan Mahler, quien señaló que las escuelas de medicina no contemplan en su formación las necesidades del pueblo ${ }^{19}$, cabe preguntarse: ¿Qué tipo de médico se está formando hoy en Chile?

Desde la lectura de la bibliografía citada se desprende que hay dos enfoques que sintetizan las principales áreas de competencias de perfiles profesionales más orientados al trabajo generalista y/o en APS: el humanista y el social. Estos conversan con el resto de las áreas de competencias, especí- ficamente clínicas y de investigación. El presente estudio identifica los discursos asociados a estos enfoques en los perfiles de egreso, contrastándolos con las recomendaciones de la OPS.

Además, Chile asumió las recomendaciones internacionales para educación superior basada en competencias, cuya base es la explicitación de perfiles de egreso, elemento central para el sistema de acreditación.

\section{Material y Método}

Investigación cualitativa con técnica de análisis documental de los textos de los perfiles de egresos declarados publicados en las páginas web de las carreras de medicina pertenecientes a ASOFAMECH. Se excluyeron las declaraciones complementarias: "descripción de la carrera" o "competencias del egresado".

Se incluyeron las siguientes universidades: Andrés Bello, de Antofagasta, Austral de Chile, Católica del Norte, Católica de la Santísima Concepción, de Chile, del Desarrollo, Diego Portales, Finis Terrae, de la Frontera, de Concepción, Mayor, Pontificia Universidad Católica de Chile, San Sebastián y de Valparaíso (Universidad de los Andes, Católica del Maule y de Santiago de Chile no tenían perfiles publicados a octubre de 2013).

Se realizó análisis de contenido "ciego", vale decir las unidades de estudio fueron codificadas. Se utilizó el software Atlas Ti 6.0.

Mediante una sistematización emergente se obtuvieron las siguientes categorías y subcategorías de análisis (Tabla 1).

\section{Resultados}

Los hallazgos del estudio se muestran en la Tabla 2, "Matriz de análisis de los perfiles de egreso según categorías y subcategorías y adecuación a las recomendaciones de OPS" para cada Universidad, segregados según su consideración respecto a las orientaciones de la OPS. En la Figura se especifica la inclusión de cada subcategoría con cruces.

El primer campo temático abordado es el Enfoque Social. En este eje fueron encontrados elementos referidos a un nivel elevado de abstracción, como el pensamiento crítico y los de- 
Tabla 1. Categorías y subcategorías de análisis

\begin{tabular}{|c|c|}
\hline Categorías & Subcategorías: consideración y/o incorporación de \\
\hline Enfoque social & $\begin{array}{l}\text { - Desarrollo de pensamiento crítico } \\
\text { - } \text { - Cultura } \\
\text { - Salud comunitaria } \\
\text { - } \text { Familia } \\
\text { - Objetivos sanitarios y/o de la política de salud } \\
\text { - Modelo bio-psicosocial } \\
\text { - Promoción y/o prevención } \\
\text { - Trabajo en APS y/o nivel primario } \\
\text { - Aspectos docente/pedagógicos }\end{array}$ \\
\hline Enfoque humanista & $\begin{array}{l}\text { - Humanización de la atención } \\
\text { - Formación ética y/o en ética } \\
\text { - Valores religiosos }\end{array}$ \\
\hline Habilidades socio/comunicacionales & $\begin{array}{l}\text { - } \text { Trabajo en equipo } \\
\text { - } \text { Rol de liderazgo } \\
\text { - Gestión y administración } \\
\text { - Adaptación a los cambios } \\
\text { - Autonomía, autoaprendizaje y educación continua } \\
\text { - Habilidades comunicacionales }\end{array}$ \\
\hline Orientación profesional & $\begin{array}{l}\text { - Orientación hacia la especialización } \\
\text { - Perfil generalista }\end{array}$ \\
\hline
\end{tabular}

terminantes sociales, así como también el trabajo en atención primaria y los aspectos pedagógicos de la profesión. Además, se incluyeron todos los aspectos que apuntaban a la cultura, la salud comunitaria y la familia. Se verificó la existencia de menciones hacia los objetivos sanitarios, también las relativas al modelo biosicosocial, y a la promoción y prevención en salud. Una universidad presentó ausencia absoluta de discurso referido al Enfoque Social, y sólo en 5 planteles se llega a una presencia de discurso por sobre la mitad de las subcategorías.

En cuanto al Enfoque Humanista, en él se vincularon los elementos referidos a los aspectos valóricos de la formación médica; la humanización de la atención, consideraciones éticas, y los valores religiosos.

En referencia a las Habilidades Socio/comunicacionales, se consideró las alusiones hacia temas como el trabajo en equipo, la capacidad de liderazgo, y la formación en aspectos administrativos. También se integraron aquellas habilidades que fomentaban la adaptación a los cambios, la autonomía, el autoaprendizaje y la actualización médica, así como los aspectos comunicacionales. Se puede observar que hay un perfil que incorpora todas las subcategorías y en cinco se abordan la mayoría de ellas. Sin embargo, hay dos universidades que no se refieren a ninguna habilidad de este tipo, y en dos casos sólo hay una única alusión a ellas.

La cuarta categoría apunta a la Orientación Profesional. Un primer hallazgo es que hay cuatro casas de estudio en las cuales no existe una orientación profesional manifiesta. Diez tienen un perfil explícitamente generalista y en seis casos se manifiesta formar a los egresados con orientación hacia la especialización profesional. De estas últimas hay una Universidad que sólo abordó esta orientación, sin incluir al perfil generalista. Hay cinco universidades que mencionan sólo la orientación profesional general, y en otros cinco casos coexisten la orientación generalista con la de especialización. 


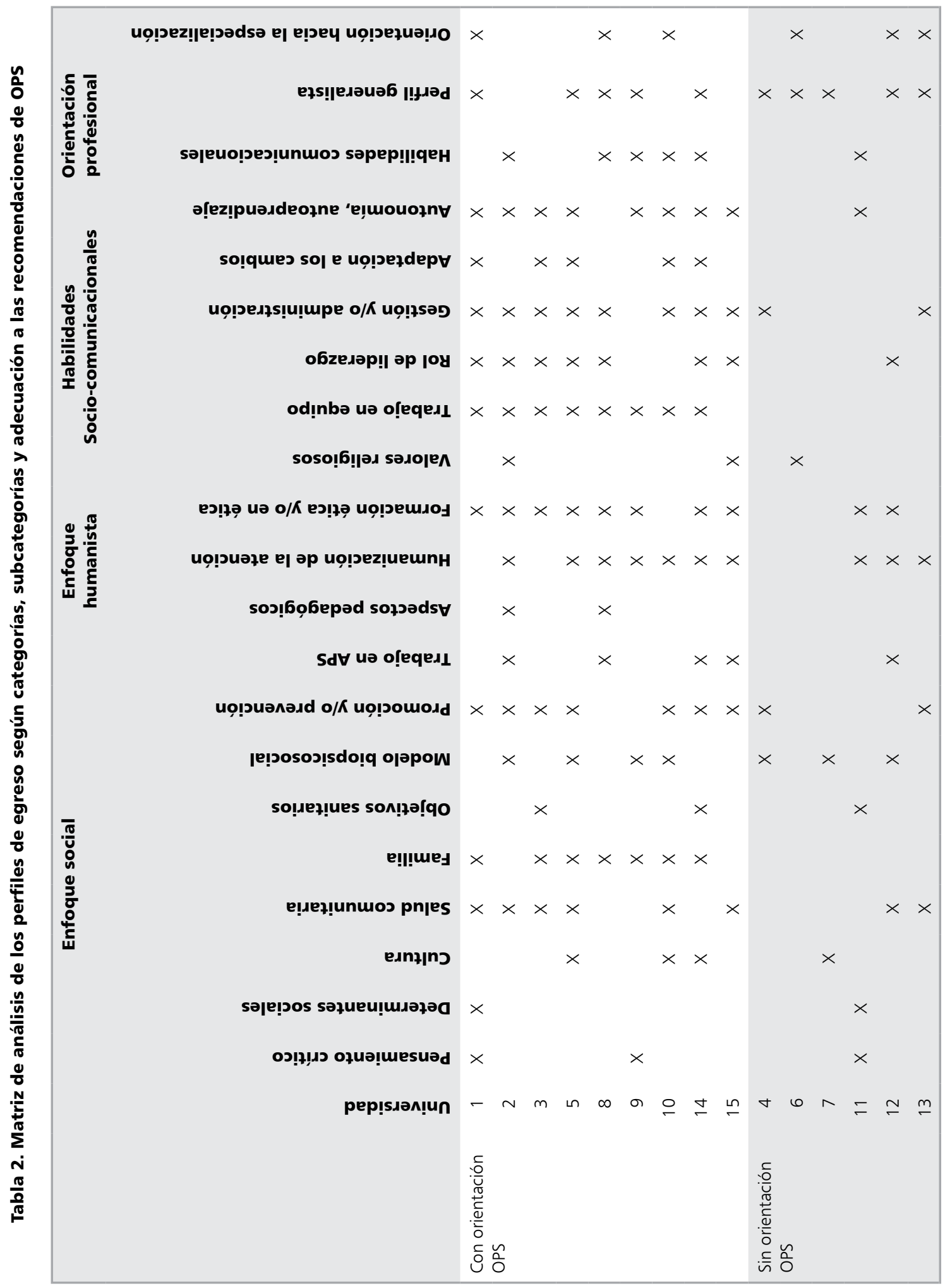




\section{Discusión}

En relación a la presencia de los elementos del Enfoque Social, es muy valorable que los aspectos asociados a promoción y prevención de la salud, junto con la consideración de la comunidad, estén muy presentes en los textos de los perfiles, ya que esto apunta hacia una visión más generalista, a la vez que preocupada más allá de lo curativo y rehabilitador, quitando centralidad a los análisis puramente individualistas de la salud-enfermedad-atención.

Pese a ello, la mayor parte de los perfiles no está considerando a la política pública, ni los objetivos sanitarios, como un eje movilizador de su formación, lo cual genera una gran duda respecto a la articulación de la educación médica con las necesidades de salud del país ${ }^{18}$.

La mínima incorporación de los determinantes sociales y del pensamiento crítico podría estar evidenciando que el resto de los elementos presentes del Enfoque Social se estén implementando sin un marco teórico referencial que los articule, tal como es recomendado actualmente por la OPS ${ }^{18,19,22}$.

La escasez de discurso respecto del trabajo en atención primaria resulta aún más inquietante si consideramos que es el foco de la estrategia de salud $^{16,18,19,22}$. En esta categoría es notoria además, la ausencia de discurso en torno a los procesos socio-históricos en los que se enmarcan las transformaciones a la protección social de la salud en el país. Estas falencias se pueden traducir en una formación médica aislada de los procesos de cambio socio-sanitario.

Los elementos del enfoque humanista se encuentran mayoritariamente incluidos en los perfiles de egreso de las universidades estudiadas, el que en algunas universidades es incluido desde la visión religiosa.

Los elementos del enfoque humanista se encuentran mayoritariamente incluidos en los perfiles de egreso de las universidades estudiadas. Sin embargo, algunas universidades lo incluyeron desde la visión religiosa.

En esta categoría, la valoración altruista presente en los perfiles, escapa en algunos casos, al contexto asistencial y se extrapola a características propias del médico en cuanto persona.

Sin embargo, estos discursos no apuntan específicamente a un trato ético, que en opinión de los autores, no parece tener como trasfondo el enfoque de derechos humanos y/o de la salud, sino que apuntan hacia una actitud compasiva con el paciente.

Es relevante que en un tercio de las casas de estudio no se encuentren contenidos referentes a la formación ética y la humanización de la atención, ya que señalan un aspecto central de la profesión médica muy exigido en la actualidad ${ }^{14,18,22}$.

$\mathrm{Al}$ analizar la presencia de discurso en ambos enfoques, los elementos del Enfoque Social son menos frecuentes que los del Enfoque Humanista. Lo anterior es coherente con la formación médica de raigambre hipocrática, que tiene mucho contenido puesto en el altruismo de la profesión y en el principio de la no maleficencia, y escasa preocupación por lo comunitario y social ${ }^{17,20}$.

Respecto de las habilidades socio-comunicacionales, encontramos que las subcategorías más mencionadas apuntan al enfoque tradicional de formación médica, vale decir, la autonomía, el autoaprendizaje, la inquietud por mantenerse permanentemente actualizado, junto con el rol de liderazgo del médico.

Las habilidades de gestión y administración están constantemente presentes en los perfiles, lo cual es coherente con la necesidad surgida durante las dos últimas décadas de que los médicos tengan una mayor preparación en esta área.

Se vislumbra en un nivel general la participación del médico al interior de los equipos de trabajo como un aspecto positivo. Esto constituye un gran soporte si se considera el desafío asumido por la APS renovada ${ }^{18,19,22}$. Sin embargo, el hecho de que la adaptación a los cambios y el trabajo en equipo no estén presentes mayoritariamente en los perfiles, es contradictorio con lo anterior.

Los elementos de las habilidades socio-comunicacionales están parcialmente incorporadas, y dirigidas al ámbito asistencial. Como este tipo de habilidades no se enfocan hacia los aspectos sociales y humanísticos de la medicina, se genera una duda razonable respecto de que dichos aspectos estén débilmente implementados en las mallas curriculares.

En cuanto a la orientación profesional, encontramos que existían universidades que explicitaron un perfil exclusivamente generalista ${ }^{18,19,22}$. Llama la atención que algunas de estas universidades incorporan el concepto de médico "integral", que de acuerdo al texto completo del perfil se utiliza como un sinónimo de "general". Existe un caso 
en donde la explicitación es sólo de orientación hacia la especialización. Sin embargo, el contenido completo de dicho perfil refiere hacia la formación de un médico general tal como en las otras. Todo apunta a que en este caso hay una omisión de redacción en el texto.

Encontramos cinco casos en donde se explici$\tan$ ambas orientaciones, situación que se condice con el contexto socio-sanitario chileno, en donde existe de facto una alta tasa de especialización precoz y una alta demanda pública de este tipo de profesionales ${ }^{25-27}$.

Es posible concluir que existe una notoria heterogeneidad en la información declarada en los Perfiles. Esta situación puede responder, entre otros factores (diferentes momentos en la redacción y/o actualización de los perfiles, consistencia con los contenidos del EUNACOM), a la desregulación que presenta el país en cuanto a la oferta en recursos humanos para salud, y particularmente respecto a las características elementales que se necesitan de los futuros médicos, cuestión atribuible a la inexistencia de una política pública potente en Educación Médica.

A partir de esta constatación, que debe ser tomada con cautela, es posible explicar aspectos que son declarados con escasa claridad en los Perfiles; en primer término, el hecho de que en ellos sólo parcialmente se declara formar a un médico general, y más aún, en algunos de estos casos dicha orientación coexiste con alusiones que apuntan a la futura especialización de los egresados. Por otra parte, es igualmente preocupante que los discursos en torno al trabajo en la Atención Primaria no sean un elemento común, teniendo en cuenta la reforma de salud y las recomendaciones de la OPS.

El Enfoque Social está presente en los discursos, pero con menos relevancia que el Enfoque Humanista. Las habilidades socio-comunicacionales no avanzan hacia una mejor relación con la comunidad y con los equipos de salud. Todo lo anterior denota incoherencia en la arquitectura argumentativa de los Perfiles en general, abriendo una serie de cuestionamientos sobre de la implementación efectiva de los enunciados que se señalan.

El estudio muestra que, al analizar cualitativamente los Perfiles de Egreso, la mayoría de las universidades chilenas no están orientando su formación médica hacia la promoción y ejercicio de una Atención Primaria Renovada, tal como han sido las indicaciones de la Organización Panamericana de la Salud.

Es preciso advertir que una limitación de la investigación es que el análisis de los datos no incorporó las diferencias entre universidades públicas y privadas, sin embargo, tiene la potencialidad de contribuir a una línea de base para el fortalecimiento del diseño, implementación y evaluación de políticas públicas al respecto.

El presente estudio debe ser complementado con investigaciones que profundicen en el grado de coherencia entre los perfiles, los planes de estudio declarados con la actividad formativa real, y con los resultados de los procesos de acreditación. Además, resulta importante conocer la voz de los actores involucrados en el proceso enseñanza-aprendizaje respecto de qué tipo de médico se está formando, cuáles son las proyecciones laborales más buscadas, cómo se realiza la formación específica en los enfoques sociales y humanistas, etc. Un aspecto relevante, no tocado en este estudio, es la problematización e investigación respecto de la pertinencia y coherencia de la formación de especialistas en medicina familiar, la política del modelo de salud integral familiar y las recomendaciones de los organismos internacionales.

Finalmente, se requiere promover un diálogo con el Ministerio de Salud para discutir la necesidad de formar médicos con visión de APS como una forma eficiente de invertir los recursos y aumentar la cobertura de salud, tal como lo requiere Chile y lo señala la OPS.

\section{Referencias}

1. Harden R. Approaches to curriculum planning. Rev Medical Education 1986; 20 (5): 458-66.

2. Harden R. Curriculum planning and Development. En a practical guide for medical teacher. Tercera edición. Churchill Livingstone-Elsevier; 2009.

3. Bloom S. Medical education in transition: paradigm change and organizational status. In RD M, ED J, editors. Medical education in transition. Princeton: The Rober-Wood Johns Foundation; 1992. p. 15-25.

4. Byrne N, Rozental M. Tendencias actuales de la educación médica y la propuesta de orientación para la educación médica en América Latina. Educ Med Salud 1994; 28 (1): 53-93.

5. Duarte E. Ciencias sociales y salud en América Latina. 
Primera edición ed. Duarte E, editor. Washington DC: OPS/CIESCU; 1986.

6. Goic A, Martínez F. Análisis del plan de estudio de las carreras de medicina. Rev Med Chile 1976; 104 (1): 104-12.

7. Oyarzún F. Perspectiva antropológica en la formación médica: experiencia de la Universidad Austral de Chile. Rev Med Chile 1994; 122 (12): 1428-9.

8. Goic A. Un nuevo currículo para la Escuela de Medicina de la Universidad de Chile. Cuad Med Soc 1995; 36 (número extraordinario): 34-6.

9. Rosselot E. Reorganización en la educación médica. Aspectos esenciales de la reestructuración de la Facultad de Medicina de la Universidad de Chile. Rev Med Chile 1997; 125 (7): 783-91.

10. Montoya C. Aspectos de la formación médica en Chile. Su relación con los conceptos de medicina integral y medicina familiar y con la planificación de recursos humanos para el sistema de salud. Cuad Med Soc 1998; 39 (2): 42-50.

11. Copaja D. Formación médica, integración de varias discipinas. Pediatria al día 1998; 16 (2): 100-6.

12. Mateluna E. Cambio curricular en el plan de estudios de la carrera de medicina de la Universdiad de Chile. Cuad Med Soc 1998; 39 (2): 51-6.

13. Jadresic A. La reforma de 1968 en la Facultad de Medicina de la Universidad de Chile. Cuad Med Soc 2008; 48 (3): 192-203.

14. Goic A, Florenzano R, Velasco C. Análisis de la formación humanística y psicosocial en el pre-grado de la carrera de medicina. Rev Med Chile 1985; 113 (5): 453-62.

15. Parada M, Gregoire J, Casanova D, Vidales A. Incorporación de contenidos de Ciencias Sociales y Humanas en el currículum de Medicina de la Universidad de Valparaíso. Cuad Med Soc 2009; 49 (4): 238-45.

16. MINSAL. Ley 19.937, sobre Autoridad Sanitaria y Gestión. [Sitio en internet]. Portal web de la Biblioteca del Congreso Nacional. Hallado en: www.bcn.cl (Acceso el 13 de septiembre de 2013).

17. Academia Chilena de Medicina. Informe sobre la situación actual de la educación médica en Chile. Serie monografías académicas Santiago: Academia de medicina del Instituto de Chile; 2008.

18. OPS. La formación en medicina orientada hacia la atención primaria de salud. Serie La renovación de la atención Primaria d de Salud en las Américas. [Sitio en internet]. Portal web de la Organización Panamericana de la Salud. Hallado en: http://new.paho.org/hq/dmdocuments/2010/APS-Formacion_Medicina_Orientada (Acceso el 2 de agosto de 2013).
19. OPS. Educación Médica hacia la APS renovada y libros de texto. Informe de la reunión de Buenos Aires, Argentina. Rosa Borrel y Rodolfo Kauffman (relatores). [Sitio en internet]. Portal web de la Organización Panamericana de la Salud. Hallado en: http://www.observatoriorh. org/?q=node/523 (Acceso el 2 de septiembre de 2013).

20. Godue C, Borrel R. Ideas principales de la conferencia de Beyond Flexner: Misión social de la Educación Médica. Tulsa, Oklahoma. [Sitio en internet] Observatorio Regional de Recursos Humanos en Salud. Hallado en: http://www.observatoriorh.org/?q=node/391 (Acceso el 2 de septiembre de 2013).

21. Godue C, Borrell R. La Acreditación de Programas de Formación Médica y la Orientación hacia la APS. Serie $\mathrm{N}^{\circ} 3 \mathrm{La}$ Renovación de la Atención Primaria en las Américas. [Sitio en internet]. Observatorio Regional de Recursos Humanos en Salud. Hallado en: http://www. paho.org/hq/index.php?option=com_content\&view=category\&layout=blog\&id=3176\&Itemid=3536\&lang=es (Acceso el 3 de septiembre de 2013).

22. Borrell R. Relato de un diálogo virtual exitoso en el portal del Observatorio de Recursos Humanos de Salud de la OPS/OMS. [Sitio en internet]. Observatorio Regional de Recursos Humanos en Salud. Hallado en: www.observatoriorh.org (Acceso el 3 de septiembre de 2013).

23. Bastías S, Marshall R, Zufina P. Número de médicos en Chile: estimaciones, proyecciones y comparación internacional. Rev Med Chile 2000; 128 (10): 1167-76.

24. Montero J, Muñoz F, Rosselot E, Valdivieso V, Barba R. La medicina general en la organización médica actual. Rev Med Chile 1996; 124 (8): 1006-14.

25. Román O, Acuña M, Señoret M. Disponibilidad de médicos en Chile al año 2004. Rev Med Chile 2006; 134 (8): 1057-64.

26. Breinbauer H, Fromm G, Fleck D, Araya L. Tendencia en el estudiante de medicina a ejercer como médico especialista. Rev Med Chile 2009; 137 (7): 865-72.

27. Román O, Pineda S, Señoret M. Perfil y número de médicos generales que requiere el país. Rev Med Chile 2007; 135 (9): 1209-15.

28. Facultad de Ciencias Médicas de la Universidad de Santiago de Chile. El médico formado en la USACH. Rev Clínica y Ciencia 2002; 1 (5): 49-57.

29. Parada M, McColl P, Barros M, González R. Una estrategia integral para mejorar la educación médica en la Universidad de Valparaíso. Universidad de Valparaíso; 2007.

30. Goic A. Seminario sobre formación de médicos en la actualidad en Chile. Rev Med Chile 2003; 131 (2): 20912. 
31. Hebel E. Presentación de la Universidad de la Frontera. [Sitio en internet]. Observatorio Regional de Recursos Humanos en Salud. Hallado en: www.observatoriorh. org (Acceso el 5 de septiembre de 2013).

32. Molina C. El médico para Chile en el siglo XXI. Cuad Med Soc 1995; 36 (Extraordinario): 37-42.

33. Bass C. ¿Cuál es el médico que necesita nuestro país?
[Sitio en internet]. El quinto poder. Hallado en: http:// www.elquintopoder.cl/salud/cual-es-el-medico-que-necesita-nuestro-pais/ (Acceso el 22 de diciembre de 2013). 34. ASOFAMECH. Reseña histórica de ASOFAMECH. [Sitio en internet]. Asociación de Facultades de Medicina de Chile. Hallado en: www.asofamech.cl (Acceso el 2 de diciembre de 2013). 